UDC 811.131.1:929 Stipčević N.

930.85(497.11:450)

DOI 10.18485/italbg.2015.2.2

Francesco Bruni*

Università di Venezia, Ca' Foscari; Accademia dei Lincei

\title{
CONTRIBUTO PARZIALE PER NIKŠA STIPČEVIĆ, ITALIANISTA $^{* *}$
}

\begin{abstract}
Degli studi italianistici pubblicati da Stipčević in lingua italiana, l'articolo presente prende in considerazione tre argomenti: il contributo dello studioso al pensiero critico ed estetico di Antonio Gramsci in rapporto all'idealismo di Benedetto Croce e alle diverse interpretazioni della critica di Francesco De Sanctis da parte di Croce e dello stesso Gramsci; il ruolo avuto in Dalmazia e in Serbia dal pensiero di Niccolò Tommaseo e Giuseppe Mazzini, intellettuale impegnato il primo, agitatore e cospiratore politico il secondo, entrambi precoci scopritori delle nazionalità slave e portatori di un pensiero che mira alla liberazione di tutte le nazionalità; infine, la riflessione (e il significato di questa riflessione) di Ivo Andrić sui Ricordi di Francesco Guicciardini, rimasta inedita ma documentata nelle carte dello scrittore, che lavorò sul moralista fiorentino negli anni nei quali viveva in una Belgrado sotto l'occupazione nazista prima, e poi sotto i bombardamenti alleati.

Parole chiave: Nikša Stipčević, Antonio Gramsci, Giuseppe Mazzini, Ivo Andrić, Francesco Guicciardini, relazioni intellettuali tra Serbia e Italia
\end{abstract}

In questo mi pare consista d'ogni nazione la vera grandezza; conservare modestamente e fermamente l'indole propria, le altre sorelle con rispettoso affetto abbracciare. Chi troppo ammira sé stesso, troppo prende da altrui [...] chi d'una nazione estera invaghisce in eccesso, risica disconoscere di quella medesima i pregi veri e deturparli con imitazione schiava. [...] Giova pertanto [...] far che i vincoli tra popolo e popolo sieno spirituali il più che ci è dato, $\mathrm{e}$ stretti da nobile affetto. Meglio che trapiantare, giova sovente innestare; che per tal modo s'ha il nuovo, e non si abbatte l'antico... (Tommaseo, Scintille 2008: 5-6)

*francescobruni1943@gmail.com

** Il contributo riprende gli spunti della relazione pronunciata al convegno di studi Le riflessioni italo-serbe. L'eredità di Nikša Stipčević tenutosi all'Accademia Serba delle Scienze e delle Arti, a Belgrado, il 3 novembre 2014. 
1. Un italianista italiano è generalmente uno studioso che raramente conosce le lingue slave, e chi scrive non fa purtroppo eccezione alla regola. Perciò questo è un contributo che si limita a prendere in esame una parte dei lavori italianistici di Nikša Stipčević pubblicati in lingua italiana, e non considera gli studi in lingua serba ${ }^{1}$. Di fatto, non prendo in considerazione se non tre centri d'interesse dell'opera dello studioso: Gramsci, Tommaseo e Mazzini, Guicciardini (e Andrić).

2. La prima opera che uno studioso italiano collega al nome di Stipčević è il libro su Gramsci e i problemi letterari (ricavato dalla sua tesi di dottorato) che uscì in serbo-croato nel 1967, e in traduzione italiana (di Sergio Turconi) nel 1968, con due nuove edizioni nel 1974 e nel 1981 (Stipčević 1981).

Antonio Gramsci (1891-1937) fu tra i fondatori, nel 1921, del Partito Comunista Italiano, nato per scissione dal Partito Socialista Italiano. Dopo una lunga lotta politica, che lo portò a scontrarsi duramente con il fascismo, Gramsci fu arrestato nel 1926 e condannato al carcere per le sue attività; fu liberato solo nel 1934, quando le sue condizioni di salute erano ormai compromesse irrimediabilmente. In carcere cominciò una seconda vita per l'accanito attivista che, in una situazione difficilissima, trovò nello studio e nella riflessione la forza per resistere alla disperazione, nonostante la solitudine affettiva, l'isolamento politico, lo stato della salute, da sempre precario, che la vita in carcere non poteva che aggravare (fino alla morte prematura, all'età di 46 anni). Come si sa, i Quaderni del carcere furono pubblicati postumi, nel secondo dopoguerra, tra il 1948 e il 1951 (Gramsci 1948-51), e su questa edizione lavorò Stipčević; successivamente uscì una nuova edizione, ordinata diversamente e commentata (Gramsci 1975).

Il lavoro su Gramsci fu presentato da Stipčević nel 1966 come tesi di dottorato, e l'anno seguente la tesi era diventata un libro, pubblicato in serbo nel 1967. Vale la pena di ricordare che, in un lavoro non dedicato a Gramsci, Stipčević osservò occasionalmente che nell'allora Jugoslavia "il nome di Gramsci cominciò ad essere citato con una certa frequenza soltanto all'inizio degli anni sessanta" (Stipčević 1989: 130), e cioè quando avviava la sua tesi di dottorato sull'intellettuale comunista.

La traduzione italiana della monografia non si fece attendere, e l'opera del giovane italianista di origine dalmata (era nato a Spalato nel 1929), formatosi nella Facoltà di Filosofia dell'Università di Belgrado, fu accolta nella prestigiosa collana "Civiltà letteraria del Novecento", dell'editore Mursia, diretta dall'italianista Giovanni Getto e condiretta dai suoi allievi

${ }^{1}$ Naturalmente mi sono servito dell'utile bibliografia delle pubblicazioni di Stipčević curata da Zogović e Milinković (2005). Ringrazio Snežana Milinković per avermi fornito vari estratti e fotocopie di articoli di Stipčević. 
Giorgio Bàrberi Squarotti ed Edoardo Sanguineti: uscito nel 1968 con il titolo Gramsci e i problemi letterari, il libro ebbe, come si è detto, un'ampia diffusione, attestata dalle ristampe. Un rapido riscontro nel Sistema Bibliotecario Nazionale (SBN) italiano, accessibile liberamente in Rete, permette di constatare che l'opera è presente in ben 110 biblioteche italiane, maggiori e minori: la cifra è da intendersi per difetto, e testimonia il buon esito dell'opera.

La pubblicazione italiana del libro su Gramsci veniva incontro, nel clima culturale di quel tempo, a istanze e interessi diversi: per il Partito Comunista Italiano Gramsci era senza dubbio il maggiore intellettuale-politico apparso in Italia, quello che meglio si poteva opporre alla cultura "borghese". La rivendicazione era giusta, e solo va aggiunto che i tanti libri usciti in quegli anni non andarono oltre le posizioni di Gramsci (e più tardi emerse un atteggiamento di rifiuto, ma non di approfondimento della sua linea di pensiero e di ricostruzione storica), mentre il suo pensiero avrebbe dovuto essere sviluppato, corretto, approfondito, aggiornato a situazioni nuove e in tal modo reso davvero vitale e operante. Senza entrare nella questione dei rapporti tra i comunisti italiani emigrati (anche nella Russia di Stalin: tra essi Palmiro Togliatti) e il prigioniero di Turi (in provincia di Bari), nel secondo dopoguerra il pensiero di Gramsci fu di grande importanza perché, nonostante l'approfondimento insufficiente e il mancato superamento dialettico cui si è accennato, contribuì ad assicurare al Partito Comunista Italiano un prestigio anche intellettuale che mancò invece ad altri partiti dell'Europa occidentale (per esempio, ai comunisti francesi).

In carcere Gramsci aveva fatto seriamente i conti con il pensiero idealistico, particolarmente con quello di Croce, il filosofo, storico e critico che, nonostante la sua posizione dichiaratamente antifascista (e forse, in parte, grazie anche a questa posizione), aveva continuato a esercitare un'influenza vastissima sulla cultura italiana fra le due guerre, filosofica e ancor più storica e critico-letteraria. Nel secondo dopoguerra, poi, molti idealisti, di osservanza sia crociana sia gentiliana, avevano compiuto una migrazione culturale e soprattutto politica passando sotto le bandiere del P.C.I.: lasciando da parte altri motivi contingenti, più o meno nobili (la convinzione sincera; l'opportunismo; l'adesione a una nuova corrente politico-culturale molto influente anche se non maggioritaria elettoralmente; l'ostilità alla religione soprattutto nella forma, di gran lunga prevalente in Italia, del cristianesimo cattolico), è noto che la dialettica hegeliana era il denominatore comune delle filosofie neoidealistiche non meno che delle diverse correnti marxiste sviluppatesi alla fine del XIX secolo e nella prima metà del XX.

Gramsci, poi, nella sua revisione critica del pensiero di Croce, ne era stato inevitabilmente condizionato, rimanendo più volte all'interno di quel sistema crociano che cercava, ora riuscendovi ora no, di superare per una 
visione più valida. Grande merito di Gramsci, tuttavia, era l'aver sottratto il marxismo italiano (almeno nella sua ala più sensibile culturalmente) al determinismo meccanicistico, di marca positivista, che aveva influenzato pesantemente varie correnti del marxismo in Europa. Nella prospettiva di Gramsci lo sviluppo dei modi di produzione economica restava il motore più importante, ma non l'unico, della dialettica storico-sociale. La convinzione che la struttura economica della società fosse più importante delle sovrastrutture giuridiche, filosofiche, religiose, letterarie e così via non era superata, ma almeno si ammetteva che se l'economia era il fattore decisivo delle dinamiche sociali e politiche, ciò era solo in ultima analisi. Questa formula era per la verità piuttosto evasiva, non cercava di individuare l'interazione di forze diverse nel concreto sviluppo storico, ma almeno in parte sottraeva il marxismo all'ipoteca di una concezione deterministica della realtà.

Anche prescindendo dalla storia politico-culturale interna al PCI, era in corso negli anni Cinquanta e Sessanta una revisione (si chiamava così) da parte della critica letteraria che cercava di superare (altra parola corrente all'epoca) il pensiero di Croce, ora cercando ispirazione nel marxismo (ma restando sostanzialmente interna al crocianesimo) ora in altre direzioni (ma, tranne qualche eccezione, con risultati non troppo diversi). Nella narrativa, poi (e nel cinema neorealista), il problema del realismo (e della denuncia sociale) era molto attuale, e si può ricordare almeno la polemica nata quando uscì (1955) il Metello di Vasco Pratolini. Queste discussioni, che implicavano una teoria e una relazione della teoria con l'esercizio della critica letteraria, vivevano, negli studi più consapevoli, in una cornice aperta agli apporti internazionali: era l'antico problema, già aristotelico, dell'arte nel suo rapporto con la realtà (la mimèsi), il problema della realtà rappresenta$t a^{2}$. Un critico marxista autorevole, in Italia e altrove, era György Lukàcs, ungherese, che anche lui interveniva, fra l'altro, sul concetto del realismo e sulla critica del romanzo. In questo clima culturale e politico, insomma, Gramsci era un punto di riferimento importante, e il libro di Stipčević usciva al momento giusto, ed era l'opera di uno studioso proveniente dalla Jugoslavia, un paese un po' meno soggetto a vincoli censori, rispetto agli stati che facevano parte del blocco sovietico.

\footnotetext{
${ }^{2}$ Così suona, alla lettera, il sottotitolo del classico libro di Erich Auerbach: Mimesis. Dargestellte Wirklichkeit in der abendländischen Literatur (1956), tradotto in italiano: Mimesis. Il realismo nella letteratura occidentale. Più vicine all'originale, ma non del tutto aderenti, le traduzioni spagnola, portoghese e inglese: Mimesis. La representación de la realidad en la literatura occidental; Mimesis. A representação da realidad na literatura ocidental; Mimesis. The Representation of the reality in Western Literature.
} 
Sullo sfondo di questa situazione s'innesta poi la fase di transizione verso una nuova sensibilità e nuove correnti politiche, che si riflette sulla ricerca e sui sistemi scolastici (Università comprese): come si è detto, la traduzione italiana è del 1968, l'anno che ha dato il nome al Sessantotto e a una contestazione partita dagli studenti universitari statunitensi ed europei che ha avuto varie facce, durate, influenze nei paesi che l'hanno conosciuta. Continuando a ricordare in maniera molto sintetica situazioni e movimenti ormai consegnati al passato (e però in modi diversi attivi nel presente), la contestazione è stato un movimento fortemente orientato a sinistra, e ha rilanciato il marxismo e il comunismo (e altre correnti) in molte versioni, diverse e spesso avverse tra loro. Ne ha beneficiato anche Gramsci, benché non siano mancate critiche e rifiuti di un pensatore che è stato definito riduttivamente troppo moderato, e insufficiente dal punto di vista di una concezione puramente materialistica, e troppo umanista di fronte ad altre concezioni che si contendevano il campo dell'ideologia negli anni Settanta.

Tornando al libro di Stipčević, studioso proveniente dalla Jugoslavia, paese socialista che nel 1948 era uscito dal blocco sovietico, era uno dei tre soci fondatori dei paesi non allineati, e sperimentava l'autogestione ma era lontano da una democrazia nel senso corrente in Occidente nel secolo scorso, egli trovava forse in Gramsci un autore che faceva parte del canone dei pensatori e dei politici socialisti-comunisti, e tuttavia, come si è detto, era fedele a un'idea della dialettica non irrigidita nella dogmatica ufficiale imperante nella vulgata vigente nei paesi dell'Europa dell'Est. In Jugoslavia insomma c'era un margine di libertà intellettuale in più, se è permessa una caratterizzazione sommaria che dovrebbe essere precisata di volta in volta sull'arco di vari decenni; ed è ovvio che la storia dei paesi dell'Europa orientale durante il sistema dell'Unione Sovietica non si lascia ricondurre a un binario unico.

Stipčević, italianista, non s'impegna direttamente sul terreno della politica, della filosofia e della dialettica o del marxismo. Tuttavia il suo Gramsci e i problemi letterari rivela uno studioso interessato alle dimensioni teoriche non solo dell'estetica e dei metodi della critica letteraria, ma della filosofia senza aggettivi. Del resto, non sarebbe stato possibile affrontare la teoria della letteratura nella prospettiva marxista di Gramsci senza fare seriamente i conti, oltre che con gli sparsi spunti di Marx sull'arte, con la generale posizione filosofica di Marx e del marxismo da un lato, e dall'altro con l'estetica e la critica di Croce (strettamente connesse con il suo sistema filosofico) e dell'idealismo, su su fino a Hegel. Stipčević mostra una solida conoscenza di questi argomenti, cosa che gli permette una chiara trattazione di Gramsci come un anti-Croce. Nella sua trattazione non c'è però un rifiuto pregiudiziale dell'idealismo, coerentemente con l'impostazione 
gramsciana che è alla ricerca di una cultura la quale sappia tener conto del meglio prodotto da un intellettuale "borghese" come Croce. Stipčević si dimostra addentro al clima culturale italiano (andando oltre la conoscenza della bibliografia specifica richiesta dall'argomento del suo lavoro) e, inoltre, è al corrente della cultura e della critica francese, come lasciano intendere alcuni riferimenti pertinenti (Stipčević 1981: 52, 53, 60 n. 26, 83). A questo proposito va ricordato che Stipčević fu lettore di serbocroato alla Sorbona dal 1959 al 1961.

Nell'ambiente culturale italiano di quegli anni, che si è descritto in termini necessariamente sintetici, si cercava il precursore di un concetto realistico della letteratura in Francesco De Sanctis (1817-1883). Era ricorrente la parola d'ordine del ritorno a De Sanctis, che la critica di sinistra o "progressista" tendeva ad annettersi in alternativa alla lettura idealistica con cui Croce aveva postulato una linea continua da De Sanctis al neoidealismo e cioè prima di tutto a sé stesso. De Sanctis è largamente presente nei Quaderni del carcere e nel libro di Stipčević, che a Gramsci e De Sanctis dedica un capitolo (De Sanctis è considerato anche altrove, per esempio nel capitolo Gramsci su Dante: Stipčević 1981: 169-181 e 147-168). Notevole, poi, è il rilievo dedicato a Gramsci su Pirandello: l'autore prende in esame gli articoli di Gramsci negli anni della militanza politica, violentemente negativi sul teatro di Pirandello, e il tono più tranquillo delle critiche, accompagnate da riconoscimenti positivi, nei Quaderni del carcere. Dall'analisi risultano l'evoluzione e le oscillazioni di giudizio di Gramsci nei confronti dell'arte drammatica pirandelliana (Stipčević 1968: 89-146).

Benché dichiari di occuparsi di "cultura e letteratura", sicché la politica dovrebbe restar fuori del discorso sull'arte e la critica, la filosofia (e con essa la politica) è ben presente, anche se sullo sfondo; il libro, poi, mostra familiarità con critici letterari come Natalino Sapegno e Luigi Russo, e inoltre con storici della filosofia come Eugenio Garin o filosofi politici come Norberto Bobbio: segno di un represso interesse filosofico slittato su meno scottanti questioni di estetica e di critica letteraria? Mi limito a porre la domanda.

Altro visibile retroscena del libro è, se non sbaglio, la consapevolezza che Gramsci sta a Croce come Marx sta a Hegel, una consapevolezza che va oltre ciò che si richiede a una trattazione limitata alla letteratura e alla critica letteraria.

Su Gramsci Stipčević è tornato con articoli e interventi congressuali: ricordo almeno la sua partecipazione al più importante convegno dedicato a Gramsci dall'Istituto che ne porta il nome, e che era, all'epoca, un'emanazione del PCI. In sede di discussione Stipčević è autore di un intervento, riprodotto negli Atti del Convegno, sulla relazione tenuta da Natalino Sapegno (Stipčević 1970: I 279-283). 
Oggi, a circa mezzo secolo di distanza dall'uscita del libro, la fase culturale segnata dal tempestivo libro di Stipčević è, inevitabilmente, lontana (come sono lontane le direzioni della teoria e della prassi critica italiana, e della politica, degli anni Cinquanta-Sessanta e oltre), e la fortuna di Gramsci continua, specialmente fuori d'Italia, verso direzioni di natura non solo politica, ma antropologica, e in una chiave libertaria.

3. Giuseppe Mazzini (Genova 1805-1872), ideatore di un movimento politico nuovo e instancabile cospiratore, e Niccolò Tommaseo (Sebenico 1802-1874), intellettuale la cui diretta militanza politica è circoscritta al ruolo di primo piano svolto nell'insurrezione antiaustriaca di Venezia nel 1848-49, ma informatissimo e pieno di idee anche in materia politica, e lungo tutto l'arco della vita impegnato in una pubblicistica civile di alto valore morale e intellettuale, sono due personalità molto diverse fra loro. Tutti e due, però, sono repubblicani (e, per questo e per altri motivi, vivranno con delusione l'approdo al Regno d'Italia nel 1861) ed entrambi (cosa più importante per il problema che qui interessa) hanno in comune un'idea della patria italiana non in termini puramente nazionali (e già questa, nella prima metà del XIX secolo, sarebbe stata un'idea audace e pericolosa per chi se ne fosse convinto e l'avesse espressa in pubblico) ma internazionali (di fatto, europei), in una visione politica che mira a nazioni indipendenti e dotate di una loro caratterizzazione, e però in un regime di concordia e di reciproco arricchimento culturale: quel fine, insomma, che l'Unione Europea dei nostri giorni sembra non solo incapace di perseguire, ma anche di concepire in termini teorici, priva com'è di un progetto e di un'idea politica all'altezza dei nostri tempi (un aspetto, evidentemente, non privo di conseguenze sul piano degli indirizzi complessivi dell'Unione stessa).

Come si sa, fu la Grecia che, nell'Europa della Restaurazione, dette il segnale del risveglio indipendentistico, ribellandosi alla dominazione ottomana e giungendo, attraverso le travagliate vicende degli anni Venti del XIX secolo, alla nascita di un piccolo stato. L'entusiasmo dei Filelleni europei era pronto a riversarsi in favore di altre nazionalità oppresse, anche se prive dell'aureola della Grecia classica e perciò estranee al mito greco. Fu così che quando Varsavia si ribellò, nel 1830, alla dominazione russa (la Polonia era scomparsa dalla carta politica dell'Europa), Tommaseo intervenne prontamente a favore dei polacchi, e nell'opera Dell'Italia, pubblicata nel 1835 a Parigi (dove era andato in esilio volontario), polemizzò aspramente contro il papa Gregorio XVI, che aveva invitato i polacchi alla rassegnazione (Tommaseo 2003: I. 48ss.). Cattolico, e convinto che la religione fosse il più solido fondamento della libertà, Tommaseo giudicava molto severamente un papa che non esortava i polacchi a liberarsi da una dominazione straniera (per di più i Russi erano di confessione ortodossa), e che non si adoperava perché le potenze dell'Europa occidentale andassero in 
soccorso della Polonia. Il papa, da parte sua, sperava che un atteggiamento conciliante avrebbe reso meno dure le condizioni dei cattolici polacchi sotto la dominazione russa (scopo, peraltro, non raggiunto).

Giuseppe Mazzini, poi, fondatore nel 1831 della Giovine Italia, dopo un fallito tentativo insurrezionale in Savoia dovette rifugiarsi in Svizzera dove, nel 1834, fondò una Giovine Europa, a conferma del reciproco rafforzamento, in lui come in Tommaseo, del patriottismo italiano e del nazionalismo plurale: un ideale molto diverso dai nazionalismi particolaristici (e talora aggressivi) che sono il frutto, parzialmente inevitabile, degli ideali della prima metà del XIX secolo. La Giovine Europa nacque a Berna il 15 aprile 1834, e il suo Atto di fratellanza fu sottoscritto da 6 italiani, 6 tedeschi, 5 polacchi.

Più tardi anche gli slavi del Sud s'imposero all'attenzione e alla coscienza di questi spiriti in anticipo sui tempi, e Mazzini, nelle Lettere slave del 1857, si chiederà:

“Chi pensava agli Slavi vent'anni addietro? Chi ravvisava, anche in fatti di tanto rilievo quanto l'insurrezione polacca del 1830, una scintilla del foco comune? Solo Napoleone, tra le grandi guerre europee, intravvide un istante l'importanza di quell'elemento..." (Mazzini 1939: II 596).

Secondo Mazzini il problema slavo era stato poco o nulla avvertito dai potenti; invece, Tommaseo e lui stesso, come si è visto, erano stati precoci nell'intuire che il generale sommovimento non era circoscritto all'Europa occidentale. Mazzini e la diffusione delle sue idee politiche ha attirato l'attenzione di Stipčević in un articolo (Stipčević 2000a) nel quale si sofferma sulla penetrazione del credo mazziniano in Serbia. Poiché la diffusione è concomitante alla trasformazione, dipendente dalle diverse strutture, sociali e culturali, del luogo di arrivo rispetto al punto di partenza, con intelligenza storica Stipčević rileva che l'esiguità del ceto medio in Serbia impedì processi politici analoghi a quelli verificatisi in Italia, sicché "il mazzinianesimo in Serbia rimase per quel momento un'utopia, ma contribuì a creare una profonda radice di affetto verso l'Italia, che [...] permane tuttora" (Stipčević 2000a: 12).

Alla Dalmazia e agli Slavi del Sud, dopo le pagine sulla ribellione polacca nel Dell'Italia, Tommaseo si volse a partire dal 1839, e mentre nel 1861 nasceva il Regno d'Italia, il problema gli si pose in termini nuovi e impegnativi, di fronte ai quali non arretrò (Pirjevec 1977). In questa sede, tuttavia, mi soffermo su un'opera molto anteriore che, per quanto non sia molto nota, è un vero e proprio classico, letterario morale politico, della letteratura europea di quegli anni, e unico nella letteratura non solo italiana: le Scintille. È un manifesto che annuncia e affianca l'impresa, realizzata in soli due anni (1841-42), consistente nella pubblicazione, in quattro volumi, 
dei Canti popolari Toscani, Corsi, Illirici, Greci (presso lo stesso editore, veneziano, delle Scintille).

Il libro è in italiano, latino, francese, greco moderno e avrebbe dovuto contenere alcune prose in illirico (cioè nello slavo del Sud); i pezzi illirici, però, furono vietati dalla censura. Nelle Scintille Tommaseo, facendo leva tanto sulla poesia popolare quanto sui classici della letteratura, svolge un discorso critico e letterario coerente con il concetto del nazionalismo plurale. Le Scintille rispecchiano in gran parte la sua biografia personale (non in tutto, come insegna la presenza della Grecia, dove Tommaseo sarebbe andato esule più tardi, scegliendo Corfù per il suo secondo esilio, dopo quello francese) $)^{3}$. La concordia tra nazioni libere fondate sulla giustizia dovrebbe produrre un arricchimento spirituale, oltre che economico, prospettiva che Tommaseo svolge con argomenti espressi in un modo affettuoso che vuole muovere $\mathrm{i}$ sentimenti, e che però si fonda anche su salde premesse razionali

I testi illirici della raccolta, soppressi dalla censura veneziana, apparvero a stampa, fuori del libro d'insieme (le Scintille) che avrebbe dovuto accoglierli, con il titolo di Iskrice, a Zagabria (1844 e 1848), a Belgrado (1845) e a Zara (1849). Dopo la svolta del 1848-49 cambiarono le condizioni (e le idee) politiche e, per limitarci alle Iskrice, altre riedizioni apparse nella seconda metà del XIX secolo (e anche oltre) sono caratterizzate da un'annessione etnico-culturale in senso croato o serbo, con un'attualizzazione molto lontana dalle intenzioni originarie di Tommaseo ${ }^{4}$. A questo proposito va osservato (con Ivetic 2014: 13-15) che l'applicazione di categorie politiche successive al momento in cui Tommaseo compose le Iskrice viene superata, da parte serba, grazie a Stipčević così come, da parte croata, per merito di Mate Zorić. Infatti, dalla storia della ricezione critica di Tommaseo in Serbia ricostruita da Stipčević si ricava che, nell'ultimo trentennio del XIX secolo e nel primo trentennio del secolo seguente, la storiografia serba considerò Tommaseo come un autore suo proprio (Stipčević 2000b).

3. Concludo con un saggio di Stipčević (1989), molto bello e di grande interesse, su Ivo Andrić e Francesco Guicciardini.

Delle carte di Andrić custodite oggi presso l'Accademia Serba di Scienze e Arti in Belgrado Stipčević ha studiato alcuni fascicoli su Guicciardini e i suoi Ricordi. Va osservato che questa parola non è rivolta solo al passato, come nell'italiano di oggi: anticamente includeva infatti, insieme con l'esperienza e le riflessioni che rielaborano l'esperienza, anche il significato di 'avvertimenti' rivolti al futuro, regole di vita. Così avviene in tanti libri di famiglia, numerosi particolarmente, ma non soltanto, a Firenze, e destinati ai discendenti e al loro ammaestramento. Guicciardini non

\footnotetext{
${ }^{3}$ Rimando a Bruni (2004) e a Tommaseo (2008).

${ }^{4}$ Sulla storia editoriale e politico-culturale delle Iskrice cfr. Ivetic (2014).
} 
scrisse i Ricordi per pubblicarli in forma di libro a stampa; la raccolta era destinata a lui e a una circolazione privata, ristretta alla famiglia. Di fatto, i Ricordi furono destinati, almeno nell'immediato, al loro autore, che non aveva figli maschi da educare.

Il lavoro di Stipčević consiste nella ricostruzione degli studi di Andrić su Guicciardini, in vista di una scelta dei Ricordi che intendeva pubblicare, tradotta e introdotta. L'edizione non fu poi realizzata, ma resta larga traccia delle letture preparatorie dello scrittore, e di appunti e riflessioni stimolate dal contatto con il grande storico e moralista fiorentino.

Stipčević svolge il lavoro con cura, indica quale edizione italiana di Guicciardini sia stata usata dallo scrittore, le biblioteche accademiche cui ha avuto accesso a Belgrado e il loro ordinamento, diverso da quello degli anni successivi; in qualche caso trova una traccia indiziaria che certi testi sono stati letti da Andrić e, probabilmente, solo da lui (Stipčević 1989: 112, n. 4).

Andrić conosceva bene parecchie lingue: non per nulla aveva avuto una lunga carriera diplomatica, come console o ambasciatore, prima a Roma presso lo Stato del Vaticano, poi a Bucarest, Trieste, Graz, Parigi, Marsiglia, Madrid e infine a Berlino, sede che abbandona nel 1941, per seguire il personale dell'ambasciata cui è stato ordinato di partire dalla Germania. Da poco i tedeschi hanno assalito la Serbia per terra e, dall'aria, con pesanti bombardamenti su Belgrado. Nella città occupata si stabilisce Andrić, ormai privato cittadino, conducendo vita ritirata. Ha già scritto Il ponte sulla Drina e Cronaca di Travnik, e durante gli anni di guerra continua a lavorare su questi romanzi e su altro, ma non pubblica nulla. Tra le attività di questo periodo silenzioso c'è l'interesse per Guicciardini. Si documenta dunque coscienziosamente sulla Firenze quattro- e cinquecentesca e sulla vita di Guicciardini, studia Machiavelli, legge con interesse Savonarola (è al corrente dell'influenza del grande predicatore su Guicciardini). Il mondo delle città stato, delle libere repubbliche italiane - i Comuni - peraltro prossime a trasformarsi in Signorie, interessa Andrić per effetto del mito di Firenze e della Toscana ma anche per una ragione più specifica: quella storia sociale e politica lo rimanda alla sponda dalmata dell'Adriatico, all'esperienza di Ragusa-Dubrovnik (Stipčević 1989: 118-119), la città stato in cui il volgare italiano è stato vitale e che nello stesso tempo ha preservato gelosamente la propria indipendenza, difesa dalla tendenza espansionistica di Venezia. Non per nulla la propensione per l'Adriatico ha fatto giungere le ricerche di Stipčević fino a Malta ${ }^{5}$.

${ }^{5} \mathrm{Su}$ questi interessi di Stipčević si veda il contributo di M. Zogović, Da L. Beccadelli alle "Composizioni in italiana favella" di Ignazio Giorgi. A margine degli studi ragusologici di N. Stipčević di prossima pubblicazione. 
La circolazione dei libri, in quegli anni difficilissimi, è ovviamente impedita, sicché Andrić, che a Guicciardini si dedica nel 1943-44, deve accontentarsi della modesta edizioncina disponibile in biblioteca e identificata da Stipčević ${ }^{6}$ (Guicciardini 1921; la copia belgradese è una ristampa del 1926). Da alcuni anni (1933) è uscita in Italia un'edizione nella quale $\mathrm{i}$ Ricordi si leggono distribuiti nelle diverse redazioni indicate dalla filologia moderna, ma il libro è inaccessibile ad Andrić (cfr. per tutto ciò Stipčević 1989: 112). L'edizione del 1921 sulla quale si basa dipende dalla prima edizione dei Ricordi, che è del 1857 e non è filologicamente accurata, anzitutto perché i Ricordi sono in numero di 403, che è la somma di redazioni diverse; perciò molti aforismi vi compaiono più volte, generalmente con variazioni; il testo dei Ricordi è preceduto da un'introduzione molto breve e accompagnato da poche, magre note storiche; quanto alle note linguistiche, sono inesistenti.

Andrić identifica i doppioni e seleziona i Ricordi da tradurre; nel suo lavoro di interpretazione letterale e intellettuale segna alcune parole di interpretazione più delicata, non tanto perché siano delle rarità, quanto per la molteplicità dei loro significati, che rende più di una volta incerta la scelta dell'accezione giusta; manifesta grande sensibilità linguistica in fatto di conoscenza dell'italiano, perché capisce che Guicciardini sa servirsi di "parole del tutto comuni", un aspetto essenziale della prosa dei Ricordi e di altri suoi scritti (non di tutti).

In una Belgrado sotto attacco (del bombardamento degli Alleati è traccia nel diario) Andrić frequenta la compagnia dell'antico scrittore fiorentino. Qualche spunto permette di intuire che cosa Andrić cercasse nel suo moralista fiorentino del XVI secolo (a torto giudicato un cinico opportunista da una tradizione critica dura a morire): ne mette in rilievo il concetto di onore, che in Guicciardini confina con l'idea della dignità, e dalla monografia su Machiavelli di Pasquale Villari, nota ad Andrić, trascrive, senza tradurlo in serbocroato, un passo significativo di Villari su Guicciardini paragonato a Machiavelli: "Alti [in Villari: altri. Una modificazione forse volontaria?] ideali, intelettuali [sic] e morali, egli non ne ha mai; quasi ne rifugge come da vane illusioni" (Stipčević 1989: 112 e n. 9). È una testimonianza disincantata (non scettica!) nella quale probabilmente Andrić si è sentito vicino a Guicciardini.

La domanda più profonda riguarda, durante gli anni bellici, la natura dell'uomo: Andrić sa che in un luogo dei Discorsi sopra la prima Deca di

${ }^{6}$ Stipčević individua la copia studiata da Andrić (Guicciardini 1921, nella ristampa del 1926), oggi presso la biblioteca della Cattedra di Italianistica della Facoltà di Filologia di Belgrado, che reca annotazioni e punti interrogativi di mano dello scrittore (Stipčević 1989: 112). 
Tito Livio (III, I) Machiavelli sostiene che i fondatori di stati repubblicani devono "presupporre tutti gli uomini rei", che 1'“animo" dell'essere umano si esprime con la "malignità"; è inoltre probabile, stando alle informazioni fornite dallo studioso, che lo scrittore conoscesse anche la critica esplicita mossa in proposito da Guicciardini a Machiavelli, nel commento al capitolo III, I dei Discorsi che si legge nelle Considerazioni sui "Discorsi" del Machiavelli scritte da Guicciardini. È chiaro perciò che pensando a Machiavelli (o anche a Machiavelli) Guicciardini ha scritto il Ricordo C 134 (secondo la numerazione moderna):

"Gli huomini tucti per natura sono inclinati più al bene che al male, né è alcuno el quale, dove altro rispecto non lo tiri in contrario, non facessi più volentieri bene che male. Ma è tanto fragile la natura degl'huomini et sì spesse nel mondo le occasione che invitano al male, che gl'huomini si lasciano facilmente deviare dal bene. Et però e savii legislatori trovorono e premii et le pene, che non fu altro che, con la speranza et col timore, volere tenere fermi gl'huomini nella inclinatione loro naturale" (Guicciardini 2009: 113).

Osserva Stipčević che, diversamente da Machiavelli, "Guicciardini è qui assai più vicino all'insegnamento cattolico verso il bene!" (Stipčević 1989: 124). Infatti la posizione di Guicciardini coincide perfettamente con l'antropologia cristiana, che dopo il peccato dei progenitori vede la natura umana rovinata ma non distrutta, e insomma capace di bene, sia pure fra tante spinte a scegliere il male. Su questo punto, dunque, sembra che ci sia convergenza tra il moralista cattolico del XVI secolo e lo scrittore agnostico del XX, che vive in tempi bui, entrambi disincantati e dotati di occhio critico, ma non per ciò radicalmente pessimisti come Machiavelli.

Vale anche la pena di citare per esteso un commento in duplice stesura, che incomincia riformulando l'inizio del Ricordo C 134 di Guicciardini. Riporto per prima la redazione verosimilmente anteriore:

“L'uomo per sé, sebbene sia in sostanza più incline al bene che al male, è un essere imperfetto (---) E siccome l'uomo, la sua vita e la sua opera, sono l'oggetto principale di tutte le nostre aspirazioni e preoccupazioni, è naturale che ad ogni passo s'incontrino difficoltà, dubbi e sempre nuove delusioni. Soltanto un'attenzione continua, una vigile cura e il freddo raziocinio [cancellato: la saggezza] possono salvarci così che non siamo abbattuti ai primi passi. Queste disposizioni non possono proteggerci dalle sofferenze e dalle difficoltà, ma ci possono insegnare come evitarle o per lo meno sopportarle più facilmente."

Più ampio il secondo commento:

'L'uomo come tale, anche se è fondamentalmente più incline al bene che al male, è un essere imperfetto, oppresso da istinti malvagi e da innumerevoli debolezze. E siccome l'uomo, e la vita nel suo insieme, come sviluppo ed azione, sono l'oggetto 
principale di tutte le nostre aspirazioni e preoccupazioni, è del tutto naturale che incontriamo ad ogni passo difficoltà, disgrazie e dubbi e sempre nuove delusioni. Soltanto un'attenzione continua, una cura vigile ed il freddo raziocinio ci possono salvaguardare perché fin dai nostri primi passi sappiamo evitare di essere ingannati, sfruttati ed abbattuti. Queste disposizioni non ci possono proteggere dalle sofferenze e dalle difficoltà, perché già per se stesse significano sofferenza, ma ci possono indicare come evitarle o almeno come sopportarle più facilmente" (Stipčević 1989: 125 e n. 27).

Andrić prende dunque le mosse dallo spunto di Guicciardini sui limiti dell'uomo. Tuttavia Guicciardini prosegue considerando l'utilità dei premi e dei castighi per evitare che prevalgano le inclinazioni negative, mentre lo scrittore di Travnik si concentra sull'individuo piuttosto che sulla società: l'imperfezione relativa è causa di insicurezza e delusione e solo un forte autocontrollo può limitare la sofferenza.

Terminata la guerra, Andrić rinunciò a pubblicare la traduzione dei Ricordi. Uscirono invece, nel 1945, Il ponte sulla Drina e la Cronaca di Travnik: due romanzi ispirati all'unità di luogo (Travnik e il ponte), portati a compimento parallelamente alle letture guicciardiniane; due romanzi che procedono con passo lento (ma non monotono), che senza commenti espliciti (i fatti, esposti con efficacia, parlano da sé) ricostruiscono ambienti e personaggi. Sono due opere d'invenzione e però la narrazione è illuminante anche per la storia di ambienti ai margini (ma non fuori) della grande storia, ne illuminano lo svolgersi lento e drammatico e scavano sentimenti e sensibilità di comunità e di individualità diverse per stirpe e religione, ebrei ortodossi cristiani di rito orientale e qualche volta di rito cattolico, chiuse e reciprocamente diffidenti, intente senza parere a sorvegliarsi sotto la temibile dominazione ottomana: quell'atmosfera affascinante e terribile dei Balcani che nessun documento storico può restituire. E nella Cronaca di Travnik l'ambiente soffocante della cittadina vede anche alle prese l'uno con l'altro, tra rari momenti di solidarietà inespressa e lo stato normale di costante rivalità, i rappresentanti ufficiali della Francia e dell'Impero asburgico, un mondo altro che entra in contatto con l'Impero ottomano e i suoi ritmi. Il tempo sembra fermo e la società statica; in realtà le cose cambiano, spesso impercettibilmente (un movimento della storia di bassa intensità), qualche volta in modo brusco, tant'è vero che nel Ponte sulla Drina si manifesta a un certo momento il funzionario asburgico che compie operazioni misteriose agli occhi dei residenti: che è un modo per rappresentare, lontano dalla storia vista dal centro, le periferie che solo a uno sguardo superficiale appaiono fuori del mondo, lontane e condannate alla banalità del quotidiano. Mi piace pensare, o azzardare, che nella Belgrado della guerra Andrić sia stato attratto dalla temperatura morale e dall'intelligenza mobile di Guicciardini, e che anche per questa via si sia salvato dalla disperazione. 
Forse, nel rispecchiamento tra teoria politica ed esemplificazione storica, antica e moderna, che è la caratteristica di Machiavelli, e ancor più nel realismo storico di Guicciardini, sorretto tuttavia dagli aforismi dei Ricordi (i quali nascono peraltro dai suoi scritti storici e politici in un continuo interscambio con le diverse redazioni dei Ricordi stessi) Andrić abbia non già imparato, ma ritrovato quell'ethos, già presente in lui, di un racconto storico oggettivo, impassibile, e però reso affascinante dall'implicita tensione umana che gli sta alle spalle, che abbia in certo modo riconosciuto per agnizione, in quei grandi storici e politici del Rinascimento, quel passo del narrare storico che aveva già appreso a regolare per suo conto.

Bisogna essere grati a Stipčević per averci fatto dono di un episodio così suggestivo e toccante, per averlo saputo ricostruire con precisione filologica, restituendone i significati con intelligenza critica.

\section{BIBLIOGRAFIA}

Bruni, F. (a cura di). (2004). Niccolò Tommaseo: popolo e nazioni. Italiani, corsi, greci, illirici (2 voll.). Roma-Padova: Antenore.

Gramsci, A. (1948-51). Quaderni del carcere (6 voll.). Torino: Einaudi.

Gramsci, A. (1975). Quaderni del carcere (Edizione critica dell'Istituto Gramsci, a cura di V. Gerratana; 4 voll.). Torino: Einaudi.

Guicciardini, F. (1921). Ricordi politici e civili. Torino: Utet.

Guicciardini, F. (2009). Ricordi (a cura di G. Palumbo). Bologna: Commissione per i testi di lingua.

Ivetic, E. (2014). L'edizione critica di "Iskrice" di Niccolò Tommaseo, nell'ambito di "Scintille". Apparato delle varianti. Italica Belgradensia, 2, 7-48.

Mazzini, G. (1939). Opere (a cura di L. Salvatorelli; 2 voll.). Milano-Roma: Rizzoli.

Pirjevec, J. (1977). Niccolò Tommaseo tra Italia e Slavia. Venezia: Marsilio.

Stipčević, N. (1970). Gramsci e i problemi della letteratura: Gramsci-Croce. In P. Rossi (a cura di), Gramsci e la cultura contemporanea. Atti del Convegno Internazionale di Studi Gramsciani, Cagliari, 23-27 aprile 1967 (2 voll.). Roma: Editori Riuniti - Istituto Gramsci.

Stipčević, N. (1981). Gramsci e i problemi letterari. Milano: Mursia.

Stipčević, N. (1989). Ivo Andrić e Francesco Guicciardini. Italica Belgradensia, 1989/2, 111-132.

Stipčević, N. (2000a). Serbia e Italia nel XIX secolo. Quaderni Giuliani di Storia, 21, 1, 7-22.

Stipčević, N. (2000b). Tommaseo e la Serbia. In R. Turchi \& A. Volpi (a cura di), Niccolò Tommaseo e Firenze (pp. 253-271). Firenze: Olschki. 
Tommaseo, N. (2003). Dell'Italia libri cinque (a cura di G. Balsamo-Crivelli; 2 voll.; rist. anast. 1920). Torino: UTET.

Tommaseo, N. (2008), Scintille (a cura di F. Bruni, con la coll. di E. Ivetic, P. Mastandrea, L. Omacini). Milano: Fondazione Pietro Bembo / Ugo Guanda Editore.

Zogović, M. \& Milinković, S. (a cura di). (2005). Curriculum vitae et studiorum [di N. Stipčević]. Italica Belgradensia (Numero speciale. Studi in onore di Nikša Stipčević), 7-28.

\section{SOME NOTES ON THE ITALIAN WORKS \\ OF NIKŠA STIPČEVIĆ}

\section{Summary}

This paper focuses on three subjects among those covered in Nikša Stipčević's writings in Italian, all dealing with the intellectual history of Italy. First, the emphasis is on Stipčević's interest in the thought of Gramsci, mainly in terms of the theory and practice of literary criticism (from Dante to Pirandello). The idealistic influence on the thought of Gramsci and Gramsci's new interpretation of Italian culture are covered; the different evaluations of Francesco De Sanctis by Croce and Gramsci are also examined. Secondly, attention is given to the influence of Niccolò Tommaseo and Giuseppe Mazzini in Dalmatia and Serbia of the $19^{\text {th }}$ century: although different in their historical role (Tommaseo being an intellectual engagé, Mazzini a conspirator), during the first half of the $19^{\text {th }}$ century they both promoted the Romantic sense of national patriotism in an international, plural and pacific perspective, and they both aimed at the liberation of all European nations from foreign domination. Finally, the focus is placed on Stipčević's analysis (based on unpublished manuscripts kept in the University Library in Belgrade) of Ivo Andrić's work on the Ricordi of the moralist Francesco Guicciardini, work conducted during the Second World War, while Andrić was living in a Nazi-occupied Belgrade.

Keywords: Nikša Stipčević, Antonio Gramsci, Giuseppe Mazzini, Ivo Andrić, Francesco Guicciardini, intellectual relations between Serbia and Italy 